\title{
Taponamiento cardiaco por perforación de la arteria coronaria tras ICP
}

\author{
Cardiac tamponade due to coronary artery perforation after PCI \\ Jonatan Vargas Caño1,*, Raquel Crespo Sainz-Aj1, Haizea Montes Hijosa1', Karmelo Intxaurraga Fernández', \\ Sorkunde Telletxea Benguria' \\ 1 Anestesiología, Reanimación y Terapéutica del dolor, Hospital Universitario Galdakao-Usansolo.
}

Financiación: La presente investigación no ha recibido ayudas específicas provenientes de agencias del sector público, sector comercial o entidades sin ánimo de lucro.

Conflicto de intereses: Los autores declaran no tener ningún conflicto de interés.

Fecha de recepción: 05 de julio de 2021 / Fecha de aceptación: 07 de agosto de 2021

\begin{abstract}
Coronary artery perforation during percutaneous coronary intervention $(\mathrm{PCI})$ is a rare complication, but it can lead, among other complications, to cardiac tamponade that can even lead to death, if there is no intervention[1]. Since there are not many published cases on acute management in a critical care unit, we present the case of a 70-year-old woman who was admitted from the Hemodynamics Service after a scheduled catheterization for an occlusion of the right coronary artery. The procedure was difficult due to the patient's vasculopathy and during it a coronary rupture was caused that required a polyurethane-coated stent implant for sealing. Although initially the patient did not present any clinical repercussions, she subsequently became hemodynamically unstable with echocardiographic data of cardiac tamponade.
\end{abstract}

Key words: Cardiac tamponade, percutaneous coronary intervention, coronary perforation.

\section{RESUMEN}

La perforación de la arteria coronaria (PC) durante una intervención coronaria percutánea (ICP) es una complicación rara, pero que puede provocar, entre otras complicaciones, un taponamiento cardíaco (TC) el cual puede conducir incluso a la muerte si no hay intervención[1]. Debido a que la publicación de casos sobre el manejo agudo en una unidad de cuidados críticos no es abundante, presentamos el caso de una mujer de 70 años que ingresó desde el Servicio de Hemodinámica tras cateterismo programado para desoclusión de la arteria coronaria derecha (CD). El procedimiento resultó dificultoso por la vasculopatía de la paciente y durante el mismo se ocasionó una rotura coronaria que precisó implante de stent recubierto de poliuretano para su sellado. Aunque inicialmente la paciente no presentó ninguna repercusión clínica, posteriormente se inestabilizó hemodinámicamente con datos ecocardiográficos de taponamiento cardiaco.

Palabras clave: Taponamiento cardiaco, intervención coronaria percutánea, perforación coronaria.

\section{Introducción}

a perforación coronaria (PC) durante la intervención coronaria percutánea (ICP) es una complicación infrecuente causada comúnmente por la salida del alambre de angioplastia, el inflado del balón o el stent o la aterectomía rotacional[2]. Puede tener mal pronóstico por el riesgo de taponamiento cardiaco (TC), formación de seudoaneurismas, arritmias, shock cardiogénico o muerte[3]. El TC se caracteriza por la acumulación de líquido pericárdico que provoca la compresión de todas las cámaras cardíacas debido al aumento de la presión pericárdica. El pericardio tiene cierto grado de elasti- 
cidad; pero una vez que se alcanza el límite elástico y, a medida que aumenta el volumen pericárdico, las cámaras cardiacas disminuyen su tamaño reduciendo la distensibilidad diastólica ${ }^{4}$. Una vez que el pericardio ya no puede estirarse, pequeños volúmenes de 50-100 ml de líquido pueden provocar una descompensación hemodinámica[5].

\section{Caso clínico}

Se presenta el caso de una mujer de 70 años que ingresó al Servicio de Reanimación, desde el Servicio de Hemodinámica, tras cateterismo programado para desoclusión de la arteria coronaria derecha (CD). Dicha programación se planteó por el diagnóstico previo de enfermedad de las arterias coronarias de 2 vasos con oclusión de la CD y lesión en arteria circunfleja (Cx), presentando, además, una insuficiencia cardiaca descompensada con función sistólica de ventrículo izquierdo (VI) deprimida y estenosis aórtica moderada-severa. Por ello, se optó por la colocación de un stent farmacoactivo (SFA) en Cx e intervención de la $C D$ en un segundo tiempo.

Como antecedentes relevantes la paciente presentaba: alergia al contraste yodado, fumadora activa importante, dislipemia mixta, EPOC enfisematoso no agudizador, oclusión de carótida interna derecha y estenosis del 50\% de carótida interna izquierda, estenosis de arteria femoral superficial izquierda intervenida mediante una endarterectomía y plastia con parche de pericárdico bovino y un stent en la arteria iliaca común izquierda.

Durante el cateterismo coronario la cateterización arterial fue dificultosa dada la vasculopatía manifiesta de la paciente, con varios intentos fallidos, logrando finalmente un abordaje por vía arterial radial izquierda. Se realizó una aterectomía rotacional y varias predilataciones con balones de 1'25, 2 y 3 $\mathrm{mm}$, lográndose posteriormente implantar dos SFA-s con buen resultado angiográfico. Para optimizar el resultado se intentó una posdilatación con un balón de 3,5 mm; sin embargo, se ocasionó una perforación coronaria que precisó implante de stent recubierto de poliuretano para sellar la rotura coronaria iatrogénica y reversión de la anticoagulación. En dicho momento, la paciente permaneció hemodinámicamente estable y sin manifestar signos de TC.

A la llegada al Servicio de Reanimación, la paciente conservó una tensión arterial media (TAM) > 70 mmHg y una frecuencia cardíaca a $90 \mathrm{lpm}$ y se mantuvo eupneica con $\mathrm{SpO}_{2}>$ 94\%. A los 5 minutos sufrió un deterioro cardiorrespiratorio con inestabilización hemodinámica, disminuyendo la TAM a 53 $\mathrm{mmHg}$ y con taquicardia de 110 lpm, y aparición de una dinámica ventilatoria no efectiva con respiración agónica, necesidad de utilizar de la musculatura accesoria, y una disminución de la $\mathrm{SpO}_{2}$ a $90 \%$. Por ello, se llevó a cabo una ecoscopia a pie de cama donde se objetivó un derrame pericárdico que condicionaba una disfunción diastólica de la aurícula y el ventrículo derecho (VD) acompañada de una dilatación de la vena cava inferior $(\mathrm{VCl})$ sin cambios de colapsabilidad a la inspiración (Figura 1). Después del diagnóstico de TC post-ICP, se llevó a cabo fluidoterapia intensiva de rescate y se realizó una pericardiocentesis de urgencia por acceso subcostal con colocación de drenaje con un débito hemático inicial de 500 ml. Además, se canalizó la vía venosa central yugular interna derecha para la

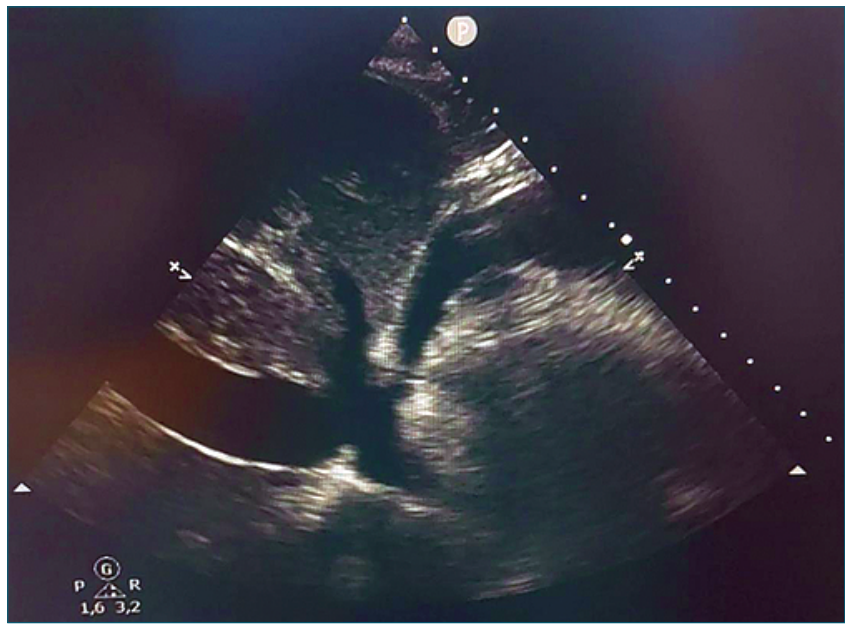

Figura 1. Colapso de VD durante la diástole y VCI dilatada sin colapsabilidad durante la inspiración.

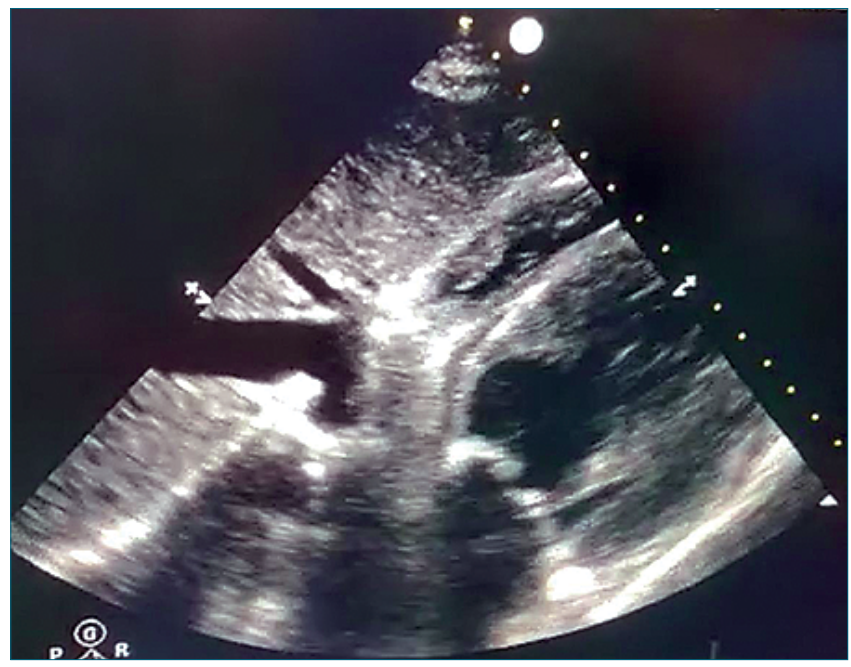

Figura 2. Aumento de llenado diastólico y disminución de dilatación de la $\mathrm{VCl}$ después de la pericardiocentesis.

administración de noradrenalina a 0,15 mcg/ $\mathrm{kg} / \mathrm{min}$. Tras dicho abordaje inicial, la paciente experimentó una optimización hemodinámica, con recuperación parcial de la función de las cámaras cardiacas derechas (Figura 2), y mejoría a nivel respiratorio. Aun así, dado que el débito hemático no se detenía, se decidió traslado al Hospital de referencia con disponibilidad de Servicio de Cirugía Cardiaca para una posible solución definitiva, dada la carencia de dicha especialidad en nuestro centro. A lo largo de su estancia en el Hospital al que se trasladó a la paciente, continuó con débito hemático abundante por el drenaje, por lo que se le realizó una nueva coronariografía, nuevamente por vía radial, que descartó fuga coronaria, y un total de 2 esternotomías por sospecha de ruptura cardiaca donde no se observó punto hemorrágico claro ni lesión en el ventrículo como posible consecuencia a la pericardiocentesis de urgencia, aunque se hallaron abundantes coágulos en mediastino y pleura derecha. Finalmente, se decidió la colocación de drenaje pleural y mediastínico desde los que se obtuvo un débito hemá- 
tico cada vez menor con la recuperación hemodinámica de la paciente y la retirada de los fármacos inotrópicos al quinto día de ingreso. Fue dada de alta del Servicio de Reanimación con un ecocardiograma reglado que revelaba una función sistólica del VI en el límite inferior de la normalidad, sin hallazgos de nuevas alteraciones segmentarias de la contractibilidad, y la estenosis aórtica moderada-severa ya conocida.

\section{Discusión}

La incidencia general de PC durante una ICP es aproximadamente del $1 \%[2]$ con aumento de dicha incidencia hasta el $9 \%$ en las ICP para oclusiones totales crónicas[6]. En la revisión realizada por Adamczyk et al.[5], la PC conduce a TC en un 11,5\%-35\%. Los factores de riesgo de perforación durante una ICP son: la edad avanzada, el sexo femenino, el uso de clopidogrel, un injerto previo de derivación de arteria coronaria, la hipertensión, presencia de enfermedad arterial periférica o insuficiencia cardiaca congestiva, índice de masa bajo y el uso de aterectomía rotacional y láser[5].

En la mayoría de los estudios de ICP, el TC se diagnosticó en el laboratorio de cateterismo cardíaco mediante ecocardiografía o fluoroscopia. Sin embargo, puede ocurrir la presentación tardía (hasta 24 h), manifestándose con hipotensión progresiva por disminución del gasto cardíaco, siendo imperativo el diagnóstico y el tratamiento para evitar un desenlace fatal[5]. La clínica de los pacientes con TC depende del tiempo durante el cual se acumula el líquido pericárdico y de su situación basal. Otras manifestaciones pueden ser la taquicardia para compensar el gasto cardiaco, la taquipnea y disnea, el dolor torácico y distensión venosa por aumento de la presión venosa yugular[4]. La mayoría de dichos signos clínicos se manifestaron en el caso expuesto.

El diagnóstico del TC, además de basarse en la historia y los signos clínicos, se puede apoyar en diferentes pruebas complementarias. El ECG puede ser útil si muestra bajos voltajes o alternancias eléctricas; una radiografía de tórax puede mostrar un aumento de la silueta cardiaca respecto a radiografía previas; y un TAC torácico también puede detectar derrame pericárdico[7]. Sin embargo, tal y como recomiendan las diferentes guías internacionales, la ecocardiografía debe ser la prueba de imagen inicial para evaluar el impacto hemodinámico de un derrame pericárdico y la guía de la pericardiocentesis. Los signos ecocardiográficos condicionados por la presencia de derrame pericárdico en el TC son: el colapso diastólico del VD, el colapso sistólico de la aurícula derecha, la dilatación y una reducción < $50 \%$ del diámetro de la VCI durante la inspiración que refleja elevación de la presión venosa central y las variaciones respiratorias en los flujos valvulares de ambos ventrículos debido a la interdependencia ventricular[4].

El tratamiento de las perforaciones coronarias puede llevarse a cabo mediante el inflado prolongado del balón, además de revertir la anticoagulación, o mediante stents de recubrimiento o embolización dependiendo del tamaño del vaso[5]. Por otra parte, el manejo inicial del TC se lleva a cabo con sueroterapia intensiva para la repleción de volumen y evitar el colapso de las cámaras cardiacas, y con soporte de fármacos inotrópicos si es preciso. Después se debe realizar una pericardiocentesis para extraer el líquido pericárdico y aumentar el volumen sistólico por descompresión extrínseca de las cámaras cardiacas[8]. El acceso subcostal, que fue el utilizado en nuestra paciente, es el punto de incisión más común. Se suele utilizar la técnica de Seldinger y se recomienda la colocación de un drenaje en espiral. Durante todo el procedimiento, se debe monitorizar con ecocardiografía la correcta colocación de la guía y del catéter[9]. La pericardiocentesis se puede realizar de forma urgente a pie de cama para estabilizar al paciente y, en caso necesario, facilitar el traslado al quirófano para realizar una ventana pericárdica o una toracotomía y pericardiotomía quirúrgica. Si el paciente requiriese ventilación mecánica hay que evitar una ventilación con presión positiva, ya que, por aumento de la presión intratorácica, produciría mayor disminución del retorno venoso, deteriorando más el gasto cardiaco. Cuando es inevitable el uso de presión positiva, se recomienda utilizar un volumen corriente bajo, frecuencias respiratorias altas y una PEEP baja[10].

Tras el procedimiento, el paciente debe ser estrechamente monitorizado para conocer la efectividad de la técnica y para detectar posibles complicaciones (hemorragia de pericardio, lesión de grandes vasos, lesión de órganos abdominales, neumotórax o arritmias). Si el débito del drenaje es abundante y tiene un nivel de hematocrito y hemoglobina similar al sanguíneo puede proceder de una cavidad cardiaca y se debería intervenir quirúrgicamente[8], incluso con apertura completa del tórax por esternotomía[10], como sucedió en el caso expuesto.

\section{Conclusión}

La PC durante una ICP puede tener complicaciones graves como el TC. Para alcanzar dicho diagnóstico es esencial conocer la clínica y los signos ecocardiográficos que lo apoyan, como la presencia de derrame pericárdico que conduce a un colapso de las cámaras cardiacas derechas y una dilatación de la VCI que no se colapsa a la inspiración. Dado que la no intervención ante un TC puede ser mortal, el manejo debe ser rápido con una fluidoterapia intensiva, la infusión de inotrópicos y, lo más importante, la realización de una pericardiocentesis ecoguiada para la optimización hemodinámica del paciente.

\section{Referencias}

1. Nagalli S, Hajouli S. Coronary Artery Perforation. In: StatPearls. Treasure Island (FL): StatPearls Publishing; July 6, 2020.

2. Guttmann OP, Jones DA, Gulati A, et al. Prevalence and outcomes of coronary artery perforation during percutaneous coronary intervention. Eurolntervention. 2017;13(5):e595-e601. Published 2017 Aug 4. https://doi.org/10.4244/EIJ-D-16-01038

3. Teis A, Fernández-Nofrerías E, Rodríguez-Leor O, et al. Perforación coronaria causada por guías intracoronarias: factores de riesgo y evolución clínica. Rev Esp Cardiol 2010;63(6):730-4. https://doi.org/10.1016/\$0300-8932(10)70166-4

4. Hoit B. Cardiac tamponade. In: Gersh B, Hoekstra J, ed. UpToDate. Waltham, Mass.: UpToDate, 2019. https://www. uptodate.com/contents/cardiactamponade? source=history widget\#H27549078

5. Adamczyk M, Wasilewski J, Niedziela J, et al. Pericardial tamponade as a complication of invasive cardiac procedures: a review of the literature. Postepy Kardiol Interwencyjnej. 2019;15(4):394- 
403. https://doi.org/10.5114/aic.2019.90213

6. Riley RF, Sapontis J, Kirtane AJ, et al. Prevalence, predictors, and health status implications of periprocedural complications during coronary chronic total occlusion angioplasty. Eurolntervention. 2018;14(11):e1199-e1206. Published 2018 Dec 7. https://doi. org/10.4244/EIJ-D-17-00976

7. Stashko E, Meer JM. Cardiac Tamponade. In: StatPearls. Treasure Island (FL): StatPearls Publishing; November 18, 2020.
8. Willner DA, Grossman SA. Pericardiocentesis. In: StatPearls. Treasure Island (FL): StatPearls Publishing; August 30, 2020.

9. Pérez-Casares A, Cesar S, Brunet-Garcia L, et al. Echocardiographic Evaluation of Pericardial Effusion and Cardiac Tamponade. Front Pediatr. 2017;5:79. Published 2017 Apr 24. https://doi. org/10.3389/fped.2017.00079

10. Miranda P, Carvajal C. Consideraciones anestésicas en el manejo del taponamiento cardiaco. Rev Chil Anest. 2010;39:85-92. 